\title{
Psychological Impact of the COVID19 Pandemic on Healthcare Employees in Abu Dhabi Health Services Company (SEHA)
}

\author{
Ahmed $\mathrm{NN}^{1}$, Al-Jasmi SA ${ }^{2}$, AlKaabi $\mathrm{RA}^{3}$, Al-Marzooqi $\mathrm{SH}^{2}$, Abuhlaiqa $\mathrm{H}^{4}$, \\ Awadalla $S^{1}$, Al-Hayyas $\mathrm{F}^{1}$, Stip E ${ }^{5}$, Ahmad $\mathrm{A}^{6 *}$ and Wanigaratne $\mathrm{S}^{7}$ \\ ${ }^{1}$ Department of Psychiatry, Abu Dhabi Health Services Company, United Arab Emirates \\ ${ }^{2}$ Department of Family Medicine, Abu Dhabi Health Services Company, United Arab Emirates \\ ${ }^{3}$ Department of Psychology, Zayed University, United Arab Emirates \\ ${ }^{4}$ Department of ENT, Abu Dhabi Health Services Company, United Arab Emirates \\ ${ }^{5}$ Department of Psychiatry, United Arab Emirates University, United Arab Emirates \\ ${ }^{6}$ Public Health Research Centre, New York University Abu Dhabi, United Arab Emirates \\ ${ }^{7}$ Research Section, National Rehabilitation Centre, United Arab Emirates
}

Research Article

Volume 4 Issue 6

Received Date: December 06, 2020

Published Date: December 16, 2020

DOI: $10.23880 /$ eij-16000172

*Corresponding author: Amar Ahmad Department of Public Health Research, New York University Abu Dhabi, Saadiyat Marina, United Arab Emirates, Tel: 971507925859; Email: asa12@nyu.edu

\section{Abstract}

Background: The Corona Virus Disease 2019 (COVID 19) outbreak initiated its course in December 2019, and on 30th January 2020, World Health Organization (WHO) declared it as a Public Health Emergency of International Concern. The virus swept the globe at an alarming speed earning its title as a Pandemic on 11 March 2020 [WHO timeline COVID-19]. Within the context of this Pandemic, health care workers have been greatly affected. This paper reports a study of Psychological impact during the peak of COVID outbreak on health care staff of Abu Dhabi Health Services Company (SEHA), UAE's largest public health sector. Methods: An email with a link to an online questionnaire was sent out to all SEHA employees, a total of 18,371. A total of 2184 responses were collected, a response rate of 11.9\%. The questionnaire consisted of the Depression and Anxiety Screening Scale (DASS-21) and basic demographic questions. The study protocol also included a dedicated psychological support helpline for staff members.

Results: The results indicate that the majority of the participants $(77.4 \%)$ were within the normal to the mild range, $9 \%$ at a moderate level, and $13.5 \%$ were within the severe to extremely severe range. Further analysis of the results showed statistically significant differences between age, gender, nationality, profession, and marital status of the respondents in levels of Depression and Anxiety. The Helpline handled 135 Calls. The nature of psychological concerns expressed on these calls and outcomes are presented.

Conclusion: Whilst the majority showed that the psychological impact was low, $22.5 \%$ were impacted and $13 \%$ of them severely. The results may reflect the effectiveness of the measures taken by the Abu Dhabi government and SEHA. The information gained by the detailed analysis of the results will be helpful to improve the response and prepare for future pandemics as well as further developing the capacity of mental health services.

Keywords: COVID-19 pandemic; Healthcare staff; Psychological Impact; Health service provision

Abbreviations: COVID 19: Corona Virus Disease 2019; WHO: World Health Organization; DASS: Depression and Anxiety Screening Scale; SARS: Severe Acute Respiratory Syndrome; GHQ-12: General Health Questionnaire 12; SEPST: SEHA Employees Psychological Support Taskforce;
PTSD: Post-Traumatic Stress Disorder.

\section{Key Messages}

- Expansion of mental health coverage for healthcare 


\section{Epidemiology International Journal}

workers.

- Provisions for employee psychological support.

- Identification of the most vulnerable section of staff.

\section{Introduction}

The world became aware of the Coronavirus disease 2019 (Covid-19) outbreak in December 2019 and the World Health Organization (WHO) declared it a global pandemic in March 2020 [1,2]. Globally there are 66,648,144 reported infections and over 1530818 deaths as of December 6th, 2020. This pandemic in its scale of mortality is compared to the Spanish flu of 1918 which is believed to have infected 500 million people and caused over 50 million deaths [3]. In recent times the Severe Acute Respiratory Syndrome (SARS) epidemic of 2002 claimed over 700 lives and the H1N1 (Bird Flu) pandemic of 2009 caused an estimated 500,000 deaths [4].

Whilst the mental health impact of the present pandemic is being currently measured by studies around the world, research into similar outbreaks in the past gives us some indication. In Canada, Nickell et al 2004 found that 29\% of their respondents scored above the threshold for the General Health Questionnaire 12 (GHQ-12) due to the SARS outbreak [5]. Maunder and colleagues in their study during the peak of the outbreak reported health care workers experienced intense emotional reactions [6]. One of the earlier studies on the mental health impact of Covid-19 from Wuhan in China, reports adverse mental health outcomes in healthcare workers [7]. A study from Singapore found that previous findings from SARS epidemic had led to better preparedness thus mitigating the impact of adverse mental health on health workers [8]. Studies published from the Gulf region on the psychological impact of Covid-19 on health care workers found an increase in stress and anxiety with a higher incidence of stress in Omani females [9] and revealed depression and anxiety are prevailing conditions among Saudi healthcare providers [10]. The UAE's rapid response appears to have a positive effect on the Covid-19 situation with UAE recording only 592 deaths among 176429, [11] who tested positive as of December 6th, 2020, thus one of the lowest mortality rates in the world. The objectives of our study were to measure the psychological impact of Covid-19 and the rapid organizational changes in service provision, on the staff of the Abu Dhabi Health Services Company (SEHA).

\section{Methodology}

The target population was health care workers in clinical (physicians, nurses, pharmacists, other allied health) and non-clinical roles (administrators, clerical staff, and maintenance workers) across all SEHA facilities.

\section{Questionnaire}

Self-administered questionnaire was emailed to all employees. The questionnaire was designed to cover information on demographic characteristics, medical history, insurance coverage for mental health care, the validated Depression, Anxiety, and Stress Scales (DASS-21) [1] and the nature of the role in the healthcare system. The Depression, Anxiety, and Stress Scale - 21 Items (DASS-21) is a set of three self-report scales designed to measure the emotional states of depression, anxiety, and stress. Each of the three DASS- 21 scales contains 7 items, divided into subscales with similar content. Scores for depression, anxiety, and stress are calculated by summing the scores for the relevant items. The DASS-21 is based on a dimensional rather than a categorical conception of psychological disorder. The assumption on which the DASS-21 development was based (and which was confirmed by the research data) is that the differences between the depression, anxiety, and stress experienced by normal subjects and clinical populations are essentially differences of degree.

\section{Recruitment}

From all SEHA hospitals and clinics, Current SEHA employees, consenting to participate, with a basic comprehension of English and use of electronic media. An email with a link to the online questionnaire was sent out to all SEHA employees on May 10th, 2020, and made available online until June 4th, 2020, with recurrent reminders via email to a total of 18371 employees. Over 2184 responses were collected when the survey link was closed $[12,13]$.

\section{Statistical Analysis}

Analyses were performed in R version 3.6.1. All applied statistical tests were two-sided, $\mathrm{p}<0.05$ were considered as statistically significant. No adjustment for multiple comparisons was made. The Depression, Anxiety and Stress scales were computed. The Depression, Anxiety, and Stress scales were categorized into normal, mild, moderate, severe, and extremely severe categories using predefined cut-off points [14]. The sociodemographic distribution was calculated in terms of contingency tables of the $\mathrm{n}(\%)$. The correspondent Cronbach's statistic $(\alpha)$ was estimated as a measure of scale reliability with a corresponding 95\% confidence interval $(95 \% \mathrm{CI})$. The Cuzick's test was performed to identify trends for Depression, Anxiety, and Stress across the five age groups 20-29, 30-39, 40-49, 5059, and 60-69. Multivariate ordinal logistic (proportional odds) regression models were fitted to determine the level of Depression, Anxiety, and Stress scales respectively. In each model, the predictors were grouped age, gender, nationality, 


\section{Epidemiology International Journal}

and marital status. The outcome was the categorized Depression, Anxiety, and Stress scale respectively.

\section{Results}

A total of 2184 health care staff members completed the survey questionnaire (response rate of $11.9 \%$ ). Of those, 1527 (69.9\%) were female, with nurses representing 887 (40.6\%), lab technicians 113 (5.2\%), Physicians 437 (20\%), Pharmacists 143 (6.5\%) and non-clinical staff 255 (11.7\%) (Table 1).

\begin{tabular}{|c|c|c|c|c|}
\hline Level/Disorder & & Depression & Anxiety & Stress \\
\hline \multirow{2}{*}{ Normal } & cut off points & $0-4$ & $0-3$ & $0-7$ \\
\hline & No. (\%) & $1442(66 \%)$ & $1375(63.0 \%)$ & $1575(72.1 \%)$ \\
\hline \multirow{2}{*}{ Mild } & cut off points & $5-6$ & $4-5$ & $8-9$ \\
\hline & No. (\%) & $225(10.3 \%)$ & $278(12.7 \%)$ & $176(8.1 \%)$ \\
\hline \multirow{2}{*}{ Moderate } & cut off points & $7-10$ & $6-7$ & $10-12$ \\
\hline & No. (\%) & $248(11.4 \%)$ & $173(7.9 \%)$ & $173(7.9 \%)$ \\
\hline \multirow{2}{*}{ Severe } & cut off points & $11-13$ & $8-9$ & $13-16$ \\
\hline & No. (\%) & $122(5.6 \%)$ & $130(6.0 \%)$ & $157(7.2 \%)$ \\
\hline \multirow{2}{*}{ Extremely severe } & cut off points & $\geq 14$ & $\geq 10$ & $\geq 17$ \\
\hline & No. (\%) & $147(6.7 \%)$ & $228(10.4 \%)$ & $103(4.7 \%)$ \\
\hline Total & & $2184(100 \%)$ & $2184(100 \%)$ & $2184(100 \%)$ \\
\hline
\end{tabular}

Table 1: Cut-off points for each of the severity categories of Normal, Mild, Moderate, Severe, and Extremely Severe and the observed number (\%) in each of the Depression, Anxiety and Stress Scale.

A majority of respondents showed normal to mild levels of scores in the DASS-21 scales, Depression 76.3\%, Anxiety $75.7 \%$, and Stress 80.2\% (average 77.4). Moderate levels of scores were shown for Depression 11.4\%, Anxiety 7.9\%, and Stress 7.9\% (Ave 9.1). Severe to extremely severe levels of scores for Depression 12.3\%, Anxiety $16.4 \%$, and Stress $11.9 \%$ (average $13.5 \%$ ) by respondents. Supplementary Table 2 shows the distribution of the 2184 health care staff members in the age groups, gender, nationality, and marital status respectively.

\begin{tabular}{|c|c|c|}
\hline Variable & Category & N (\%) \\
\hline \multirow{4}{*}{ Age } & $(20-29)$ & $207(9.5)$ \\
\cline { 2 - 3 } & $(30-39)$ & $938(42.9)$ \\
\cline { 2 - 3 } & $(40-49)$ & $684(31.3)$ \\
\cline { 2 - 3 } & $50+$ & $355(16.3)$ \\
\hline Gender & Female & $1527(69.9)$ \\
\hline \multirow{4}{*}{ Nationality } & Arab non-local & $641(29.3)$ \\
\cline { 2 - 3 } & Emirati & $382(17.5)$ \\
\cline { 2 - 3 } & Non-Arab non-local & $1161(53.2)$ \\
\hline \multirow{3}{*}{ Marital Status } & Divorced-Separated & $87(4)$ \\
\cline { 2 - 3 } & Married & $1717(78.6)$ \\
\cline { 2 - 3 } & Single & $380(17.4)$ \\
\hline
\end{tabular}

Table 2: Distribution of the 2184 health care staff members in the age groups, gender, nationality, and marital status respectively.

The Cronbach's statistic $(\alpha)$ was estimated as a measure of scale reliability with a corresponding 95\% (95\% CI). A high measurement of Cronbach's statistic $\alpha$ was observed for the Depression scale 0.912 (0.903, 0.919), Anxiety scale
$0.866(0.853,0.877)$ and Stress scale $0.925(0.919,0.931)$. A high measurement for $\alpha$ suggests that the data have relatively high internal consistency. The Cuzick's trend test shows a trend for decreased Depression (z-value $=-7.661$, p-value 


\section{Epidemiology International Journal}

$<0.0001$ ), decreased Anxiety (z-value $=-8.825, \mathrm{p}$-value $<$ 0.0001 ) and decreased Stress (z-value $=-8.053, \mathrm{p}$-value $<$ 0.0001 ) across the fife age groups.

A predefined recommended cut-off values for conventional severity labels were used to categorize the Depression, Anxiety, and Stress scale into normal, mild, moderate, severe, and extremely severe respectively (Table $2)$. The normal level was the highest frequent level in the Depression 1442 (66\%), Anxiety 1375 (63.0\%), and Stress 1575 (72.1\%) scale.

Three multivariate ordinal logistic regression models were fitted to determine the level of Depression, Anxiety, and Stress scales respectively. In each model, the predictors were grouped age, gender, nationality, and marital status (Table 3). Predicted odds ratios were computed and plotted for the three performed ordinal logistic regression models of Depression, Anxiety, and Stress respectively. The predicted odds ratios were adjusted for the age group (30-39) and gender-female. In all three OLR models, no statistically significant differences between males and females were observed. Furthermore, no statistically significant differences were observed between the age group (20-29) and age group (30-39), and statistically significant differences were observed between all other age groups and age group (3039), statistically significant differences were observed in all three models between non-Arab and Arab-non-local and Emirati respectively (Figure 1). There were only statistically significant differences observed between Pharmacists versus Nurses in the Depression and Stress models. Figure 1 shows statistically significant differences between single versus married in the Depression and Stress models.

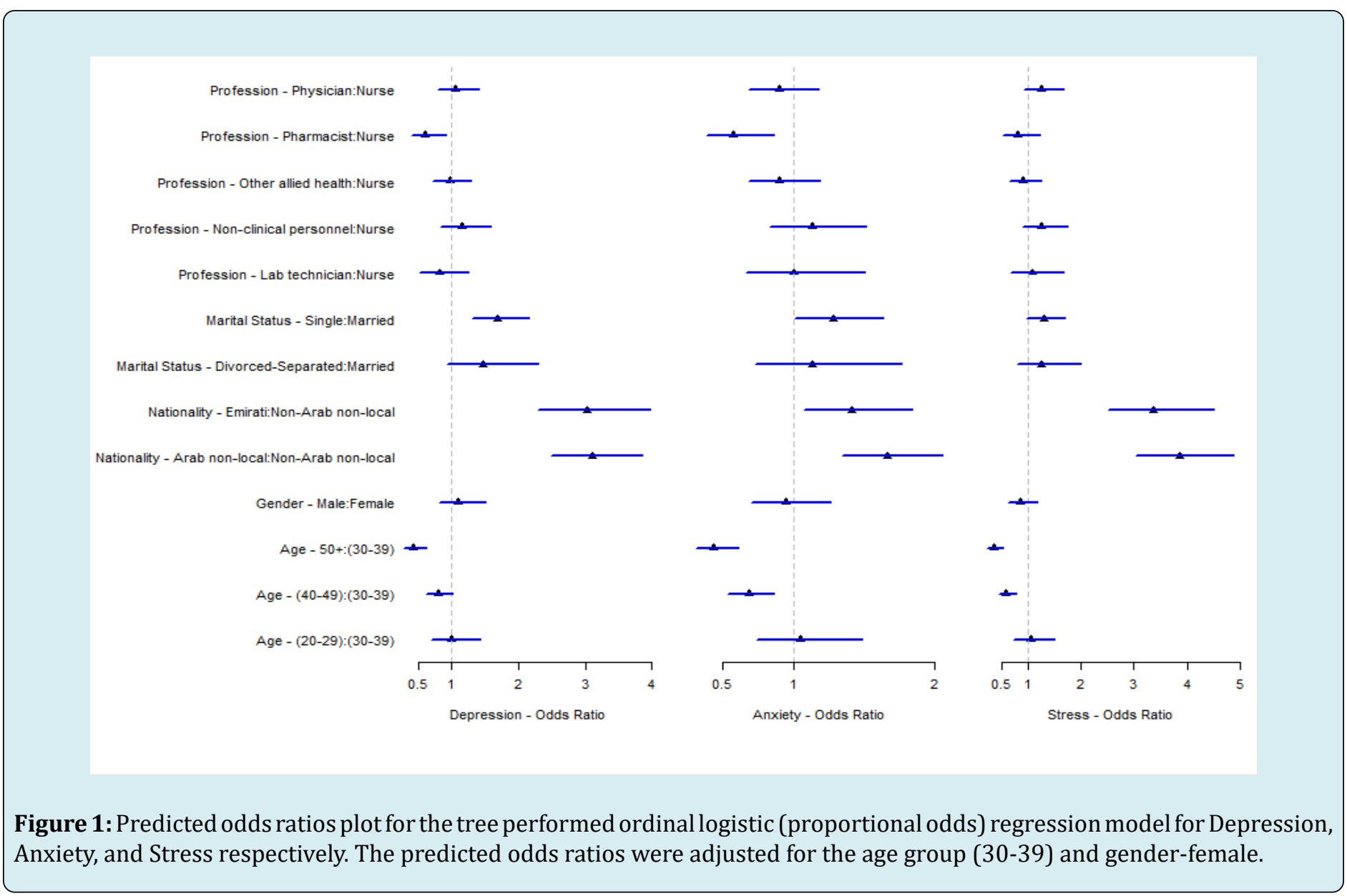


Epidemiology International Journal

\begin{tabular}{|c|c|c|c|c|c|c|}
\hline & \multicolumn{2}{|c|}{ Depression } & \multicolumn{2}{|c|}{ Anxiety } & \multicolumn{2}{|c|}{ Stress } \\
\hline & OR (95\% CI) & z-value (P) & OR (95\% CI) & z-value (P) & OR $(95 \% \mathrm{CI})$ & z-value (P) \\
\hline Age $=(20-29)$ & $1.01(0.71,1.4)$ & $0.04(0.97)$ & $1.05(0.74,1.49)$ & $0.27(0.79)$ & $\begin{array}{c}1.04(0.72 \\
1.51) \\
\end{array}$ & $0.209(0.834)$ \\
\hline Age $=(40-49)$ & $0.80(0.62,1.03)$ & $-1.73(0.08)$ & $0.68(0.54,0.87)$ & $\begin{array}{c}-3.15 \\
(0.002) \\
\end{array}$ & $\begin{array}{c}0.58(0.44, \\
0.77)\end{array}$ & $\begin{array}{c}-3.85 \\
(<0.001) \\
\end{array}$ \\
\hline Age $=50+$ & $0.43(0.30,0.63)$ & $\begin{array}{c}-4.311 \\
(<0.001)\end{array}$ & $0.43(0.31,0.61)$ & $\begin{array}{c}-4.8 \\
(<0.001) \\
\end{array}$ & $\begin{array}{c}0.35(0.23 \\
0.54)\end{array}$ & $\begin{array}{c}-4.89 \\
(<0.001) \\
\end{array}$ \\
\hline Gender $=$ Male & $1.11(0.81,1.50)$ & $0.646(0.518)$ & $0.94(0.71,1.26)$ & $-0.39(070)$ & $\begin{array}{c}0.85(0.62, \\
1.19) \\
\end{array}$ & $-0.94(0.345)$ \\
\hline Arab non-local & $3.10(2.49,3.86)$ & $\begin{array}{c}10.069 \\
(<0.001)\end{array}$ & $1.66(1.35,2.05)$ & $\begin{array}{c}4.71 \\
(<0.001)\end{array}$ & $\begin{array}{c}3.86 \text { (3.04, } \\
4.89)\end{array}$ & $\begin{array}{c}11.16 \\
(<0.001)\end{array}$ \\
\hline Emirati & $3.03(2.31,3.98)$ & $7.953(<0.001)$ & $1.41(1.07,1.84)$ & $2.48(0.01)$ & $\begin{array}{c}3.37(2.51, \\
4.52)\end{array}$ & $8.1(<0.001)$ \\
\hline Divorced-Separated & $1.48(0.95,2.30)$ & $1.730(0.084)$ & $1.13(0.73,1.76)$ & $0.55(0.59)$ & $\begin{array}{c}1.26(0.79 \\
2.00)\end{array}$ & $0.964(0.34)$ \\
\hline Single & $1.69(1.32,2.17)$ & $4.169(<0.001)$ & $1.28(1.01,1.63)$ & $\begin{array}{c}2.004 \\
(0.045) \\
\end{array}$ & $\begin{array}{c}1.29(0.99 \\
1.69) \\
\end{array}$ & $1.85(0.065)$ \\
\hline Lab technician & $0.82(0.53,1.25)$ & $-0.922(0.357)$ & $\begin{array}{c}1.003(0.67 \\
1.51)\end{array}$ & $0.01(0.99)$ & $\begin{array}{c}1.06(0.68, \\
1.67)\end{array}$ & $0.27(0.79)$ \\
\hline $\begin{array}{c}\text { Non-clinical } \\
\text { personnel }\end{array}$ & $1.16(0.85,1.58)$ & $0.931(0.352)$ & $1.13(0.84,1.52)$ & $0.78(0.43)$ & $\begin{array}{c}1.26(0.90, \\
1.76)\end{array}$ & $1.37(0.17)$ \\
\hline Other allied health & $0.97(0.73,1.30)$ & $-0.180(0.857)$ & $0.90(0.68,1.18)$ & $-0.77(0.44)$ & $\begin{array}{c}0.91(0.66 \\
1.25)\end{array}$ & $-0.59(0.56)$ \\
\hline Pharmacist & $0.61(0.41,0.92)$ & $-2.383(0.017)$ & $\begin{array}{c}0.58(0.383 \\
0.86)\end{array}$ & $\begin{array}{c}-2.68 \\
(0.007) \\
\end{array}$ & $\begin{array}{c}0.80(0.53 \\
1.22)\end{array}$ & $-1.04(0.30)$ \\
\hline Physician & $1.07(0.81,1.41)$ & $0.461(0.645)$ & $0.90(0.68,1.18)$ & $-0.79(0.43)$ & $\begin{array}{c}1.25(0.93, \\
1.68)\end{array}$ & $1.485(0.14)$ \\
\hline $\begin{array}{c}\text { Age }=(20-29) * \\
\text { Male }\end{array}$ & $0.40(0.19,0.83)$ & $-2.435(0.015)$ & $0.35(0.16,0.76)$ & $\begin{array}{c}-2.66 \\
(0.008)\end{array}$ & $\begin{array}{c}0.31(0.13 \\
0.75)\end{array}$ & $-2.59(0.01)$ \\
\hline $\begin{array}{c}\text { Age }=(40-49) * \\
\text { Male }\end{array}$ & $1.08(0.68,1.69)$ & $0.317(0.751)$ & $1.02(0.66,1.58)$ & $0.08(0.94)$ & $\begin{array}{c}1.33(0.82 \\
2.18)\end{array}$ & $1.14(0.25)$ \\
\hline Age $=50+*$ Male & $0.98(0.53,1.80)$ & $-0.074(0.941)$ & $0.56(0.30,1.06)$ & $-1.79(0.07)$ & $\begin{array}{c}0.98(0.49 \\
1.96)\end{array}$ & $-0.066(0.95)$ \\
\hline
\end{tabular}

Table 3: Results of three multivariate ordinal logistic regression models for the categorical Depression, Anxiety and Stress respectively. The category with the highest observed frequency in each variable was taken as the reference category. Odds ratio (OR) with corresponding 95\% confidence interval (95\% CI) as well as z- and p-values were computed.

\section{Data from the Staff Support Helpline}

In anticipation of psychological adverse effects on the frontline staff, SEHA Employees Psychological support taskforce (SEPST) was set up with an initiative of SEHA Telephone helpline, 'Ma'akum' or 'With you'. It was made available for all SEHA employees to call for Counselling and support at no cost. Volunteers on the helpline were psychology graduates from the community who provided telephone Counselling to staff members struggling to cope. A digital platform Takalam [13] was commissioned by SEHA to provide free of cost teleconsultation to employees (Figure 2). 


\section{Epidemiology International Journal}

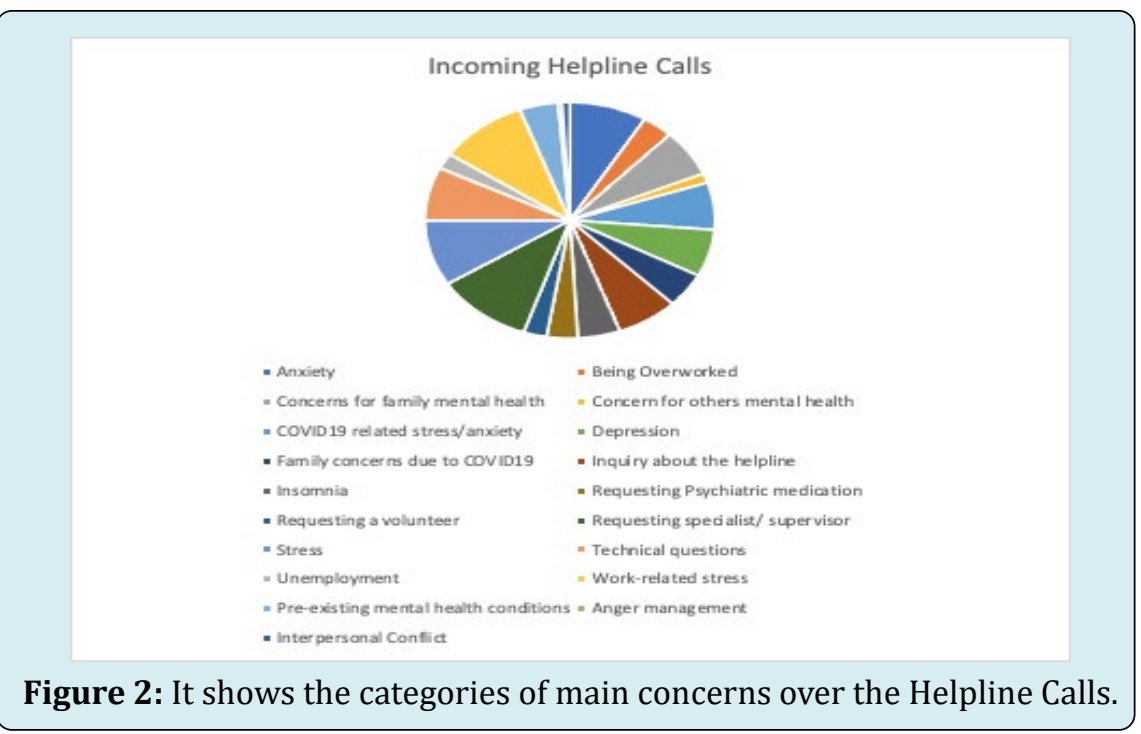

As of the $29^{\text {th }}$ of November 2020 , the helpline received 135 incoming calls. The highest portion of the calls (19\%) was requesting help from a Doctor or Specialist. $16 \%$ of calls related to general stress unrelated to Covid-19, $15 \%$ of calls were concerning different forms of anxiety, $8 \%$ of callers complained of insomnia and $11 \%$ were either depressed or experiencing depressive symptoms, which in many cases were pre-existing and elevated due to the stress current situation and inability to access needed psychiatric medications. From the calls received, 59\% needed more support with follow up sessions from a volunteer or specialist. Majority of the calls $(69 \%)$ were regarding seemingly pre-existing mental health conditions of the employee or their family members, concerns about their mental health, or being unable to get the medication that their loved one needs, resulting in worsening symptoms. Including into that the number of calls relating to the need for psychiatric medication or a specialist that increases to $94 \%$ of all calls we have received, indicating a crucial need for employees and their families to have access to mental health services.

In regard to the Covid-19 pandemic, $23 \%$ of calls have related to mental health issues and stress due to the current circumstances; $8 \%$ of which involved loneliness due to separation from family members because of travel restrictions or self-isolation, unemployment statuses (4\%), as well as unclear work protocols regarding the pandemic. Work stress (17\% of calls) were often was due to hazardous environments where regulations are not fully followed, trauma from the number of deaths seen or being overworked $(6 \%)$.

\section{Discussion}

There were several important findings from this study. It was observed that the response rate was higher amongst employees who were directly affected by the Covid-19 situation. Hence the results can be taken to be an accurate reflection of the mood state among the staff that was most psychologically impacted. The results of the DASS-21 questionnaire indicate that the majority of staff $77.4 \%$ were within the normal to the mild range, $9 \%$ at a moderate level, and $13.5 \%$ were within the severe to extremely severe range levels of depression, anxiety, and stress. This finding may indicate the UAE's preparedness and SEHA's effective implementation of pandemic plans. It may also reflect a manageable volume of patients and adequate staffing levels at the frontline facilities.

There is a lot to learn from the $22 \%$ of staff who indicate that they are impacted by the situation and the $13 \%$ severely impacted. The severely impacted figure is comparable with other studies such as Wu P, et al. [14] 2009 who found 10\% of their sample meeting the criteria for Post-Traumatic Stress Disorder (PTSD) [14]. The detailed analysis of the results as well as the qualitative data gathered from the Helpline reveals information particularly relevant to the UAE, important for developing services and targeted interventions.

Statistically, significant gender differences were found in anxiety and stress scales $(\mathrm{P}<0.0001)$ and $(\mathrm{P}<0.005)$ with females scoring higher. This may be due to females being assigned to frontline duties and having greater workloads and less support. There was a trend for single people to score higher on the three scales of the DASS-21. Physicians and non-clinical personnel scored significantly higher than other groups $(\mathrm{P}<0.0002)$. The high depression and stress scores among physicians may be an indication of workload and burnout and the non-clinical staff may have fears of exposure to the virus, have to work longer hours, and may have less job satisfaction in general. Respondents who were local nationals (Emiratis) and from other Arabic countries 


\section{Epidemiology International Journal}

scored significantly higher in all three scales compared to all other nationalities.

The qualitative data collected from the Helpline helped to create a picture of the stresses frontline staff was dealing with. The lack of insurance cover for medical help for mental health problems for the workers and their families emerged as a key theme. The second most dominant theme was the stress of infection control procedures introduced to contain the Covid-19 virus. Another theme that emerged was job insecurity as a result of the pandemic. General Covid-19 related stress, arising from what they saw as a hazardous environment where regulations were not properly followed, trauma from the amount of death seen initially, and feeling overworked and underappreciated.

\section{Limitations}

Time when the survey was disseminated and the status of the Pandemic was at its peak of the first wave world-wide. Data obtained from questionnaires was verified with medical records for validation (pre-existing psychiatric conditions). The study does not assess socioeconomic status which could influence the psychological impact.

\section{Conclusions and Recommendations}

The study captured a real-time picture of the psychological impact on SEHA staff, the largest and leading healthcare provider dealing with the Covid-19 situation. Approximately a quarter of the staff was moderately stressed and $11 \%$ experiencing severe stress. Significant relationships between demographic variables and depression, anxiety, and stress scales of DASS-21 found are of unique interest to the UAE and should help in developing interventions and services. The qualitative information gathered from the dedicated helpline set up for the study revealed a wealth of information critical to dealing with the present situation and planning for handling future pandemics. Based on our findings, we strongly recommend expansion in insurance coverage and access to mental health services for all health workers. Having a dedicated telephone helpline for psychological support as a part of the pandemic response plan. Clear guidelines to managers about workload and support, including a confidential complaint procedure. Also, measures to deal with job insecurity, and finally a longitudinal study in the aftermath of the pandemic, to assess for psychological sequelae from stressful events. There are many lessons to be learned at present, on how to deal with the current pandemic and adequately prepare for future ones.

\section{Ethics Approval}

The study was approved by the Department Abu Dhabi COVID19 Research IRB Committee. Recruitment was done among the current SEHA employees, consenting to participate, with basic comprehension of English and use of electronic media, from all SEHA hospitals and clinics. The purpose of the study was explained at the beginning of the electronic survey through a consent form inbuilt into the questionnaire. The respondent was given the opportunity to ask questions via a dedicated email address for the study. Waiver for signed consent was requested since the study presents no more than minimal risk of harm to subjects and involved no procedures. No personal identifiers were collected, and confidentiality was maintained.

\section{Acknowledgments}

We are grateful to all the healthcare staff of SEHA for their participation in this study. We would like to extend our gratitude to the Zayed university Psychology department for their interns and students who volunteered to provide Counselling to the employees through the helpline.

\section{References}

1. Lovibond S (1995) Manual for the depression anxiety stress scales. $2^{\text {nd }}(E d n)$, Sydney N.S.W, Psychology Foundation of Australia.

2. (2020) Timeline of WHO's response to COVID-19. World Health Organization.

3. (2020) Centers for Disease Control and Prevention.

4. (2020) World Health Organization.

5. Nickell LA, Crighton EJ, Tracy CS, Al Enazy H, Bolaji Y, et al. (2004) Psychosocial effects of SARS on hospital staff: Survey of a large tertiary care institution. CMAJ 170(5): 793-798.

6. Maunder RG, Lancee WJ, Rourke S, Hunter JJ, Goldbloom D, et al. (2004) Factors associated with the psychological impact of severe acute respiratory syndrome on nurses and other hospital workers in Toronto [Internet]. Vol. 66, Psychosomatic Medicine. Psychosom Med 66(6): 938942.

7. Kang L, Li Y, Hu S, Chen M, Yang C, et al. (2020) The mental health of medical workers in Wuhan, China dealing with the 2019 novel coronavirus. Lancet Psychiatry 7(3): 14.

8. Chan AOM, Chan YH (2004) Psychological impact of the 2003 severe acute respiratory syndrome outbreak on health care workers in a medium size regional general hospital in Singapore. Occup Med 54(3): 190-196.

9. Badahdah A, Khamis F, Al Mahyijari N, Al Balushi M, Al Hatmi H, et al. (2020) The mental health of health care 


\section{Epidemiology International Journal}

workers in Oman during the COVID-19 pandemic. Int J Soc Psychiatry pp: 1-6.

10. AlAteeq DA, Aljhani S, Althiyabi I, Majzoub S (2020) Mental health among healthcare providers during coronavirus disease (COVID-19) outbreak in Saudi Arabia. J Infect Public Health 13(10): 1432-1437.

11. https://doh.gov.ae/https://doh.gov.ae/
12. (2020) Abu Dhabi Health Services Company (SEHA).

13. https://www.takalam.ae/

14. Wu P, Fang Y, Guan Z, Fan B, Kong J, et al. (2009) The psychological impact of the SARS epidemic on hospital employees in China: Exposure, risk perception, and altruistic acceptance of risk. Can J Psychiatry 54(5): 302311. 